\title{
THE ROLE OF COMPUTED TOMOGRAPHIC SCANNING IN DIAGNOSING MEDIASTINAL NODE INVOLVEMENT IN NON-SMALL CELL LUNG CANCER
}

\author{
Kazuya Takamochi, MD \\ Kanji Nagai, MD \\ Junji Yoshida, MD \\ Kenji Suzuki, MD \\ Yasuhisa Ohde, MDa \\ Mituyo Nishimura, MD \\ Kenro Takahashi, MDa \\ Yutaka Nishiwaki, MD $^{\mathrm{a}}$
}

Objectives: The reliability of computed tomographic scanning in evaluating mediastinal node involvement is controversial because of the high false result rate. We attempted to identify significant factors responsible for falsepositive and false-negative scans.

Methods: From August 1992 through April 1997, 401 patients with lung cancer who underwent major lung resection and systematic lymph node dissection were enrolled in this study. We retrospectively examined mediastinal node size, tumor location, maximum tumor dimension, the presence or absence of obstructive pneumonia, atelectasis, pulmonary fibrosis, and lymph node calcification on contrast-enhanced computed tomographic scans. We identified clinical and radiologic factors responsible for the false results by using univariate and multivariable analysis.

Results: Central tumor location proved to be a significant factor of false-positive scans. Elevated carcinoembryonic antigen level and larger tumor dimension were significant factors of false-negative scans. In patients with a peripheral tumor smaller than $40 \mathrm{~mm}$ and normal levels of serum carcinoembryonic antigen, sensitivity, specificity, positive predictive value, and negative predictive value were $6 \%, 93 \%, 8 \%$, and $90 \%$, respectively. The reliability of computed tomographic scanning in this low-risk subgroup was high in detecting N0-1 disease but low in diagnosing N2 disease.

Conclusion: It is not possible to accurately diagnose N2 disease by using lymph node size on computed tomographic scanning alone, especially in patients with a central tumor, an elevated serum carcinoembryonic antigen level, or a tumor of $40 \mathrm{~mm}$ or larger. A preoperative invasive staging procedure is indicated in these populations and may not be indicated in the population with normal computed tomographic scan results without any of these risk factors. (J Thorac Cardiovasc Surg 2000;119:1135-40)
C omputed tomographic (CT) scanning is a widely used noninvasive imaging procedure for diagnos-

From the Division of Thoracic Oncology, ${ }^{\mathrm{a}}$ National Cancer Center Hospital East, Chiba, and Division of Thoracic Surgery, ${ }^{\mathrm{b}}$ National Cancer Center Hospital, Tokyo, Japan.

The study was supported in part by a Grant-in-Aid for Cancer Research from the Ministry of Health and Welfare, Japan.

Received for publication Aug 24, 1999; revisions requested Dec 1, 1999; revisions received Dec 28, 1999; accepted for publication Jan 18, 2000.

Address for reprints: Kazuya Takamochi, MD, Department of Thoracic Oncology, National Cancer Center Hospital East, 6-51, Kashiwanoha, Kashiwa, Chiba 277-8577 Japan (E-mail: ktakamoc@east.ncc.go.jp).

Copyright (C) 2000 by The American Association for Thoracic Surgery

$0022-5223 / 2000 \$ 12.00+0 \quad \mathbf{1 2} / \mathbf{1} / \mathbf{1 0 5 8 3 0}$

doi: $10.1067 / \mathrm{mtc} .2000 .105830$ ing mediastinal node involvement (N2) in patients with non-small cell lung cancer (NSCLC). However, the reliability of CT scanning in predicting N2 disease is controversial. Various results have been reported concerning the accuracy of CT scans in diagnosing N2 disease. $^{1-9}$

Dales and associates ${ }^{10}$ performed a meta-analysis of CT accuracy in diagnosing N2 disease in patients with NSCLC by using data from 42 studies published between 1980 and 1988. They concluded that the overall accuracy of mediastinal CT scanning was only $80 \%$, with approximately $20 \%$ false-positive and $20 \%$ falsenegative results. A high false-positive rate reduces specificity and the positive predictive value, whereas a high false-negative rate impairs sensitivity and the negative predictive value of an examination. The high frequency of false-positive and false-negative results sub- 
Table I. Clinical characteristics of patients

\begin{tabular}{lccc}
\hline & Overall & $F P$ & $F N$ \\
\hline No. of patients & 401 & 57 & 56 \\
Age, y (mean \pm SD) & $63 \pm 10$ & $66 \pm 9$ & $63 \pm 10$ \\
Sex (M/F) & $245 / 156$ & $46 / 11$ & $32 / 24$ \\
Smoking, pack-years (median) & 29 & 41 & 34 \\
25th-75th percentile & $(0-50)$ & $(14-55)$ & $(0-52)$ \\
$\quad$ Pack-years $>40$ & 134 & 29 & 22 \\
$\quad$ Pack-years $\leq 40$ & 267 & 28 & 34 \\
CEA, ng/mL (median) & 11.7 & 10.8 & 22.1 \\
$\quad$ 25th-75th percentile & $2.5-7.3$ & $3.1-10.0$ & $3.4-11.2$ \\
Histologic typing & & & \\
$\quad$ Adenocarcinoma & 290 & 29 & 44 \\
$\quad$ Squamous cell carcinoma & 85 & 19 & 11 \\
$\quad$ Large cell carcinoma & 15 & 3 & 0 \\
$\quad$ Adenosquamous cell carcinoma & 11 & 6 & 1
\end{tabular}

$F P$, False-positive scans; $F N$, false-negative scans; $C E A$, carcinoembryonic antigen.

stantiated the poor reliability of CT scanning in mediastinal lymph node evaluation.

Therefore, we attempted to identify clinical and radiologic factors responsible for false-positive and falsenegative results of CT scanning. We also re-evaluated the efficiency of CT scans in predicting N2 disease on the basis of lymph node size, taking these factors into account.

\section{Methods}

From August 1992 through April 1997, 634 patients with lung cancer underwent surgical intervention at our institute. Among them, 401 patients who underwent major lung resection and systematic lymph node dissection were enrolled in this study. Seven of them underwent preoperative mediastinoscopy, which revealed no mediastinal node involvement. The following patients were excluded: (1) patients who received induction therapy, (2) patients with multiple primary lung cancers, and (3) patients with a primary tumor directly invading the mediastinum. The time interval between CT scanning and surgical dissection was less than 1 month in all the patients. CT scans were done on an X-Vision/SP system (Toshiba, Tokyo, Japan), and contiguous 10-mm thick sections were obtained from the pulmonary apices to the bases in a supine position at full inspiration. Dynamic incremental scanning was always performed, and scans were nearly always obtained after bolus injection of $100 \mathrm{~mL}$ of iopamidol by using an automatic injector.

All CT scans were reviewed by 3 authors (K.T., Y.O., and K.S.), who were not informed of the surgical outcome, to obtain the following information: the maximum dimension and location of the primary lesion, the sizes of mediastinal lymph nodes, and the presence of obstructive pneumonia, atelectasis, pulmonary fibrosis, or lymph node calcification. If multiple nodes within a single station were enlarged, the size of the largest node was recorded. The location of a tumor was considered to be central when it was located in the inner one third of the lung field on CT scanning and peripheral when in the outer two thirds. Histologic typing was determined according to the World Health Organization classification. ${ }^{11}$ The stage of the disease was based on the TNM classification of the International Union Against Cancer (UICC). ${ }^{12}$ The mediastinal lymph node evaluation on CT scanning and node mapping on thoracotomy were based on Naruke's lymph node mapping. ${ }^{13}$ All resected lymph nodes were fixed in formalin and examined microscopically by means of standard hematoxylin and eosin stain. Clinical N2 disease was defined when any mediastinal lymph node was $1.0 \mathrm{~cm}$ or larger in the shortest dimension. Pathologic N2 disease was defined when any mediastinal node was histologically involved. A true-positive result was documented when a patient had both clinical $\mathrm{N} 2$ and pathologic $\mathrm{N} 2$ findings.

The clinical record of each patient was reviewed for age, sex, histologic typing, smoking status, and serum carcinoembryonic antigen (CEA) level. Serum CEA was measured by means of the two-site immunoenzymometric assay (Tosoh Inc, Yamaguchi, Japan), and the upper normal limit for this assay was $5 \mathrm{ng} / \mathrm{mL}$.

Univariate and multivariable analyses were performed by the logistic regression procedure on StatView 5.0 software (SAS Institute, Inc, Cary, NC) with a Macintosh Performa 6420 computer (Apple Computer, Cupertino, Calif) to determine the relationship between false-positive and false-negative scans and the following clinical or radiologic findings: age, sex, smoking status (pack-years $>40$ vs $\leq 40$ ), histologic typing (squamous cell carcinoma vs others), serum CEA level, tumor location, maximum tumor dimension, the presence or absence of obstructive pneumonia, atelectasis, pulmonary fibrosis, and lymph node calcification on CT scan. The variables, such as age, node, and tumor dimension, were not skewed. The serum CEA level and smoking (pack-years) were severely skewed. Thus, we used the continuous variables for age, node, tumor dimension, and the log-transformed serum CEA level. For smoking status, we used the categories of pack-years of greater than 40 versus packyears of 40 or less because the pack-years showed a two-peak distribution. The potential factors of false results in univariate analyses were also evaluated by using multivariable analyses. Differences were considered statistically significant when the $P$ value was less than .05 .

\section{Results}

The clinical characteristics of the patients are presented in Table I, and the radiologic characteristics of the tumors are presented in Table II. There were 57 (14\%) cases of false-positive scans and 56 (14\%) cases of false-negative scans.

Univariate analysis revealed 9 potential factors for false-positive scans (Table III): age, sex, smoking status, serum CEA level, histology, tumor location, obstructive pneumonia, atelectasis, and lymph node calcification. In 
Table II. Radiologic characteristics of tumors

\begin{tabular}{lccc}
\hline & Overall & $F P$ & $F N$ \\
\hline No. of patients & 401 & 57 & 56 \\
Shortest dimension of lymph node, mm (mean \pm SD) & $7 \pm 4$ & $11 \pm 2$ & $6 \pm 3$ \\
Longest dimension of lymph node, mm (mean \pm SD) & $11 \pm 6$ & $17 \pm 4$ & $11 \pm 6$ \\
Maximum tumor dimension, mm (mean \pm SD) & $32 \pm 16$ & $35 \pm 16$ & $37 \pm 17$ \\
Tumor location (central/peripheral) & $44 / 357$ & $14 / 43$ & $6 / 50$ \\
Obstructive pneumonia, $\mathrm{n}(\%)$ & $33(8)$ & $10(18)$ & $3(5)$ \\
Pulmonary fibrosis, $\mathrm{n}(\%)$ & $14(3)$ & $4(7)$ & $0(0)$ \\
Atelectasis, $\mathrm{n}(\%)$ & $14(3)$ & $5(9)$ & $1(2)$ \\
Lymph node calcification, $\mathrm{n}(\%)$ & $34(8)$ & $10(18)$ & $5(9)$ \\
\hline
\end{tabular}

$F P$, False-positive scans; $F N$, false-negative scans.

Table III. Relationship between clinicoradiologic factors and false-positive scans: Univariate analysis

\begin{tabular}{lccc}
\hline \multicolumn{1}{c}{ Variable } & OR & $95 \%$ CI & P value \\
\hline Age & 1.03 & $1.00-1.07$ & .05 \\
Sex (male vs female) & 3.05 & $1.53-6.09$ & .002 \\
Smoking (pack-years $>40$ vs $\leq 40)$ & 2.36 & $1.34-4.16$ & .003 \\
CEA $^{*}$ & 1.82 & $1.00-3.29$ & .05 \\
Histology (SCC vs others) & 2.11 & $1.14-3.89$ & .02 \\
Maximum tumor dimension (mm) & 1.01 & $0.99-1.03$ & .2 \\
Tumor location (central vs peripheral) & 3.41 & $1.68-6.93$ & .001 \\
Obstructive pneumonia & 2.97 & $1.33-6.63$ & .008 \\
Pulmonary fibrosis & 2.52 & $0.76-8.33$ & .1 \\
Atelectasis & 3.58 & $1.15-11.10$ & .03 \\
Lymph node calcification & 2.84 & $1.28-6.31$ & .01 \\
\hline
\end{tabular}

$O R$, Odds ratio; $C I$, confidence interval; $C E A$, carcinoembryonic antigen; $S C C$, squamous cell carcinoma.

"Log-transformed serum CEA levels were used.

multivariable analysis only tumor location proved to be a significant factor of false-positive scans. Lymph node calcification was a marginally significant factor $(P=.06$, Table IV). Patients with central-type lung cancer were prone to be overestimated as having $\mathrm{N} 2$ disease.

Univariate analysis revealed two potential factors of false-negative scans: serum CEA level and maximum tumor dimension on CT scan (Table V). These potential factors were also significant in multivariable analysis (Table VI).

In patients with a central tumor, the false-positive rate was $32 \%$. In patients with an elevated CEA level, the false-negative rate was $18 \%$, and in patients with a tumor $40 \mathrm{~mm}$ or larger in the maximum dimension, it was $20 \%$. The reliability of CT scanning was poor in these populations (Tables VII and VIII).

In patients with a peripheral tumor smaller than 40 $\mathrm{mm}$ and a normal serum CEA level, both the false-positive and false-negative rates were lower than those found in overall patients. Specificity and negative predictive value were extremely high $(93 \%$ and $90 \%$, respectively). Thus, the reliability of CT scanning in this low-risk subgroup was high in detecting the population without mediastinal node involvement. However, CT scanning was not reliable in diagnosing mediastinal node involvement because sensitivity and positive predictive value were extremely low $(6 \%$ and $8 \%$, respectively; Table IX).

\section{Discussion}

Mediastinal node involvement is a very important prognostic factor in patients with potentially resectable NSCLC. ${ }^{14,15}$ Several studies have suggested that induction chemotherapy or chemoradiation followed by surgical resection results in pathologic down-staging and better long-term survival in patients with $\mathrm{N} 2$ disease. ${ }^{16-18}$ Therefore, accurate preoperative evaluation of the mediastinal nodes is important in deciding NSCLC treatment strategy.

The CT scan is a routine examination for the diagnosis and locoregional staging of lung cancer at most institutes. CT scans have revealed evident reliability in 
Table IV. Relationship between clinicoradiologic factors and false-positive scans: Multivariable analysis

\begin{tabular}{lccc}
\hline \multicolumn{1}{c}{ Variable } & OR & $95 \%$ CI & P value \\
\hline Age & 1.03 & $1.00-1.07$ & .08 \\
Sex (male vs female) & 2.15 & $0.94-4.94$ & .07 \\
Smoking (pack-years $>40$ vs $\leq 40)$ & 1.08 & $0.54-2.19$ & .8 \\
CEA $^{*}$ & 1.24 & $0.63-2.44$ & .5 \\
Histology (SCC vs others) & 1.14 & $0.56-2.32$ & .7 \\
Tumor location (central vs peripheral) & 2.64 & $1.16-6.01$ & .02 \\
Obstructive pneumonia & 1.54 & $0.58-4.09$ & .4 \\
Atelectasis & 1.84 & $0.44-7.62$ & .4 \\
Lymph node calcification & 2.29 & $0.97-5.45$ & .06 \\
\hline
\end{tabular}

$O R$, Odds ratio; $C I$, confidence interval; $C E A$, carcinoembryonic antigen; $S C C$, squamous cell carcinoma.

*Log-transformed serum CEA levels were used.

Table V. Relationship between clinicoradiologic factors and false-negative scans: Univariate analysis

\begin{tabular}{lccc}
\hline \multicolumn{1}{c}{ Variable } & OR & $95 \%$ CI & P value \\
\hline Age & 1.00 & $0.97-1.03$ & .9 \\
Sex (male vs female) & 0.83 & $0.47-1.46$ & .5 \\
Smoking (pack-years >40 vs $\leq 40)$ & 1.35 & $0.75-2.41$ & .3 \\
CEA $^{*}$ & 2.02 & $1.12-3.64$ & .02 \\
Histology (SCC vs others) & 0.90 & $0.44-1.82$ & .8 \\
Maximum tumor dimension (mm) & 1.02 & $1.00-1.04$ & .03 \\
Tumor location (peripheral vs central) & 0.97 & $0.39-2.41$ & .9 \\
Obstructive pneumonia & 0.59 & $0.18-2.02$ & .4 \\
Atelectasis & 0.46 & $0.06-3.62$ & .5 \\
Lymph node calcification & 1.07 & $0.40-2.89$ & .9 \\
\hline
\end{tabular}

$O R$, Odds ratio; $C I$, confidence interval; $C E A$, carcinoembryonic antigen; $S C C$, squamous cell carcinoma.

"Log-transformed serum CEA levels were used.

Table VI. Relationship between clinicoradiologic factors and false-negative scans: Multivariable analysis

\begin{tabular}{lccc}
\hline \multicolumn{1}{c}{ Variable } & OR & $95 \%$ CI & P value \\
\hline CEA $^{*}$ & 1.92 & $1.05-3.51$ & .03 \\
$\begin{array}{l}\text { Maximum tumor } \\
\text { dimension }(\mathrm{mm})\end{array}$ & 1.02 & $1.00-1.04$ & .04 \\
\hline
\end{tabular}

$O R$, Odds ratio; $C I$, confidence interval; $C E A$, carcinoembryonic antigen. ${ }^{*}$ Log-transformed serum CEA levels were used.

examining the primary tumor in terms of size, location, and extrapulmonary extension. ${ }^{4}$ However, they are far from satisfactory and less accurate than mediastinoscopy in node involvement evaluation. ${ }^{19,20}$ Detection of lymph node metastasis on CT scan is based on the demonstration of lymph node enlargement. However, several investigators reported that benign nodes might be enlarged as a result of reactive hyperplasia, obstructive pneumonia, or atelectasis attributable to primary tumor or prior granulomatous disease, whereas metastatic nodes may appear normal in size if the metastasis is microscopic. ${ }^{5,21,22}$ These fac-
Table VII. CT scan reliability in diagnosing mediastinal node involvement: Relationship between a significant factor of FP and FPR

\begin{tabular}{lccc}
\hline & No. of cases & No. of FP scans & FPR $(\%)$ \\
\hline Tumor location & & & \\
Central & 44 & 14 & 32 \\
Peripheral & 357 & 43 & 12 \\
\hline
\end{tabular}

$F P$, False positive; $F P R$, false-positive rate.

tors can lead to false-positive or false-negative scans, which impair the reliability of CT scans in detecting node involvement. Therefore, we attempted to clarify which clinical and radiologic factors were significantly related to false results on CT scans.

Multivariable analysis revealed only one significant factor of false-positive scans: central tumor location. Mediastinal lymph nodes may be swollen reactively by obstructive pneumonia or atelectasis associated with the tumor. ${ }^{5,21}$ In our study, patients with obstructive pneumonia or atelectasis were more likely to have false-positive scans. However, once tumor location was 
Table VIII. CT scan reliability in diagnosing mediastinal node involvement: Relationship between significant factors of FN and FNR

\begin{tabular}{lccc}
\hline & $\begin{array}{c}\text { No. of } \\
\text { cases }\end{array}$ & $\begin{array}{c}\text { No. of } \\
\text { FN scans }\end{array}$ & FNR (\%) \\
\hline $\begin{array}{l}\text { Serum CEA level } \\
\quad\end{array}$ & & \\
$\quad 15 \mathrm{ng} / \mathrm{mL}$ & 173 & 31 & 18 \\
$\quad<5 \mathrm{ng} / \mathrm{mL}$ & 228 & 25 & 11 \\
$\begin{array}{l}\text { Maximum tumor dimension } \\
\geq 10 \mathrm{~mm}\end{array}$ & 397 & 56 & 14 \\
$\geq 20 \mathrm{~mm}$ & 329 & 51 & 16 \\
$\geq 30 \mathrm{~mm}$ & 195 & 32 & 16 \\
$\geq 40 \mathrm{~mm}$ & 104 & 21 & 20 \\
$\geq 50 \mathrm{~mm}$ & 50 & 12 & 24 \\
$\geq 60 \mathrm{~mm}$ & 31 & 8 & 26 \\
$\geq 70 \mathrm{~mm}$ & 16 & 4 & 25 \\
\hline
\end{tabular}

$F N$, False negative; $F N R$, false-negative rate; $C E A$, carcinoembryonic antigen.

accounted for, obstructive pneumonia or atelectasis per se did not provide additional information concerning false-positive results. Several reports compared the reliability of CT mediastinal evaluation between central and peripheral tumors. Daly and associates ${ }^{3}$ reported that CT detection of positive mediastinal lymph nodes had an accuracy of $79 \%$ for central tumors and 90\% for peripheral ones. Moreover, the false-positive rate was higher in central tumors (14\%) than in peripheral ones $(5 \%)$. Our results were generally consistent with theirs. However, our series revealed a higher falsepositive rate (32\%) in the central tumor population than that found in their series. Because these false-positive populations can benefit from primary surgery alone, an invasive staging procedure, such as mediastinoscopy, may be indicated.

Elevated serum CEA level and larger tumor dimension were significant factors of false-negative scans in both univariate and multivariable analyses. The larger a tumor has grown, the more normal-sized lymph nodes may have microscopic metastases. Some investigators reported a relationship between elevated serum CEA levels and advanced disease or poor prognosis. The patients with marked elevation of preoperative serum CEA levels are highly likely to have occult metastases. ${ }^{23-25}$ In patients with an elevated CEA level, metastatic nodes may appear normal in size. The prognosis of patients with false-negative scans for mediastinal node involvement has been reported to be poor, even though it was better than that of patients with truepositive scans. ${ }^{26,27}$ The false-negative subgroup may benefit from induction therapy, which might indicate an invasive staging procedure.
Table IX. Comparison of reliability of CT scans in diagnosing mediastinal node involvement between overall patients and patients without risk factors of false results

\begin{tabular}{lcc}
\hline & Overall & $\begin{array}{c}\text { Patients without } \\
\text { risk factors }\end{array}$ \\
\hline No. of patients & 401 & 165 \\
No. of patients with pN2 (\%) & $80(20)$ & $17(10)$ \\
Sensitivity (\%) & 30 & 6 \\
Specificity (\%) & 82 & 93 \\
Accuracy (\%) & 72 & 79 \\
PPV (\%) & 30 & 8 \\
NPV (\%) & 83 & 90 \\
FPR (\%) & 14 & 6 \\
FNR (\%) & 14 & 10 \\
\hline
\end{tabular}

$p N 2$, Pathologic N2 disease; $P P V$, positive predictive value; $N P V$, negative predictive value; $F P R$, false-positive rate; $F N R$, false-negative rate.

*Patients with a peripheral tumor smaller than $40 \mathrm{~mm}$ and a normal serum CEA level.

In patients without risk factors of false results (ie, patients with a peripheral tumor smaller than $40 \mathrm{~mm}$ and an elevated serum CEA level), CT scanning was extremely reliable in detecting the population without mediastinal node involvement. One hundred thirtyseven (90\%) of 153 patients with clinical N0-1 disease on CT scanning had pathologic N0-1 disease. Thus, patients with normal CT results in this low-risk population can proceed directly to thoracotomy. A preoperative invasive staging procedure may not be indicated. Phillips and colleagues ${ }^{28}$ reported that as prevalence of disease decreases, the reliability of a positive result will drop, and the reliability of a negative result will increase. The prevalence of $\mathrm{N} 2$ disease in our proposed low-risk subgroup of false results was extremely low, which results in low reliability of CT scanning in diagnosing mediastinal node involvement.

In conclusion, it is not possible to accurately diagnose $\mathrm{N} 2$ disease by using lymph node size on CT scan alone, especially in patients with a central tumor, an abnormal serum CEA level, or a tumor $40 \mathrm{~mm}$ or larger. A preoperative invasive staging procedure, such as mediastinoscopy, is indicated in these populations to decide the appropriate treatment strategy and may not be indicated in the population of normal CT results without any of these risk factors.

We thank Dr Satoshi Sasaki, Epidemiology and Biostatistics Division, National Cancer Center Research Institute East, for his technical supports in statistical analyses. We also thank Professor J. Patrick Barron, International Medical Communications Center, Tokyo Medical University, for reviewing the English manuscript. 


\section{REFERENCES}

1. Glazer GM, Orringer MB, Gross BH, Quint LE. The mediastinum in non-small cell lung cancer: CT-surgical correlation. AJR Am J Roentgenol 1984;142:1101-5.

2. Breyer RH, Karstaedt N, Mills SA, Johnston FR, Choplin RH, Wolfman NT, et al. Computed tomography for evaluation of mediastinal lymph nodes in lung cancer: correlation with surgical staging. Ann Thorac Surg 1984;38:215-20.

3. Daly BD Jr, Faling LJ, Bite G, Gale ME, Bankoff MS, Jung-Legg Y, et al. Mediastinal lymph node evaluation by computed tomography in lung cancer: an analysis of 345 patients grouped by TNM staging, tumor size, and tumor location. J Thorac Cardiovasc Surg 1987;94:664-72.

4. Modini C, Passariello R, Iascone C, Cicconetti F, Simonetti G, Zerilli $\mathrm{M}$, et al. TNM staging in lung cancer: role of computed tomography. J Thorac Cardiovasc Surg 1982;84:569-74.

5. Libshitz HI, McKenna RJ Jr. Mediastinal lymph node size in lung cancer. AJR Am J Roentgenol 1984;143:715-8.

6. Khan A, Gersten KC, Garvey J, Khan FA, Steinberg H. Oblique hilar tomography, computed tomography, and mediastinoscopy for prethoracotomy staging of bronchogenic carcinoma. Radiology 1985;156:295-8.

7. Martini N, Heelan R, Westcott J, Bains MS, McCormack P, Caravelli J, et al. Comparative merits of conventional, computed tomographic, and magnetic resonance imaging in assessing mediastinal involvement in surgically confirmed lung carcinoma. $\mathbf{J}$ Thorac Cardiovasc Surg 1985;90:639-48.

8. McKenna RJ Jr, Libshitz HI, Mountain CE, McMurtrey MJ. Roentgenographic evaluation of mediastinal nodes for preoperative assessment in lung cancer. Chest 1985;88:206-10.

9. Osborne DR, Korobkin M, Ravin CE, Putman CE, Wolfe WG, Sealy WC, et al. Comparison of plain radiography, conventional tomography, and computed tomography in detecting intrathoracic lymph node metastases from lung carcinoma. Radiology 1982;142:157-61.

10. Dales RE, Stark RM, Raman S. Computed tomography to stage lung cancer: approaching a controversy using meta-analysis. Am Rev Respir Dis 1990;141:1096-101.

11. The World Health Organization. Histological typing of lung tumours. Neoplasma 1982;29:111-23.

12. Mountain CF. Revisions in the International System for Staging Lung Cancer. Chest 1997;111:1710-7.

13. Naruke T, Suemasu K, Ishikawa S. Lymph node mapping and curability at various levels of metastasis in resected lung cancer. J Thorac Cardiovasc Surg 1978;76:832-9.

14. Pearson FG, DeLarue NC, Ilves R, Todd TR, Cooper JD. Significance of positive superior mediastinal nodes identified at mediastinoscopy in patients with resectable cancer of the lung. J Thorac Cardiovasc Surg 1982;83:1-11.
15. Ratto GB, Mereu C, Motta G. The prognostic significance of preoperative assessment of mediastinal lymph nodes in patients with lung cancer. Chest 1988;93:807-13.

16. Faber LP, Kittle CF, Warren WH, Bonomi PD, Taylor SGt, Reddy S, et al. Preoperative chemotherapy and irradiation for stage III non-small cell lung cancer. Ann Thorac Surg 1989;47:669-7.

17. Rosell R, Gomez-Codina J, Camps C, Maestre J, Padille J, Canto $\mathrm{A}$, et al. A randomized trial comparing preoperative chemotherapy plus surgery with surgery alone in patients with non-smallcell lung cancer. N Engl J Med 1994;330:153-8.

18. Roth JA, Atkinson EN, Fossella F, Komaki R, Bernadette Ryan M, Putnam JB Jr, et al. Long-term follow-up of patients enrolled in a randomized trial comparing perioperative chemotherapy and surgery with surgery alone in resectable stage IIIA non-small-cell lung cancer. Lung Cancer 1998;21:1-6.

19. Coughlin M, Deslauriers J, Beaulieu M, Fournier B, Piraux M, Rouleau J, et al. Role of mediastinoscopy in pretreatment staging of patients with primary lung cancer. Ann Thorac Surg 1985;40:556-60

20. Van Schil PE, Van Hee RH, Schoofs EL. The value of mediastinoscopy in preoperative staging of bronchogenic carcinoma. $\mathrm{J}$ Thorac Cardiovasc Surg 1989;97:240-4.

21. Libshitz HI, McKenna RJ Jr, Haynie TP, McMurtrey MJ, Mountain CT. Mediastinal evaluation in lung cancer. Radiology 1984;151:295-9.

22. Gross BH, Glazer GM, Orringer MB, Spizarny DL, Flint A. Bronchogenic carcinoma metastatic to normal-sized lymph nodes: frequency and significance. Radiology 1988;166:71-4.

23. Concannon JP, Dalbow MH, Hodgson SE, Headings JJ, Markopoulos E, Mitchell J, et al. Prognostic value of preoperative carcinoembryonic antigen (CEA) plasma levels in patients with bronchogenic carcinoma. Cancer 1978;42:1477-83.

24. Rubins JB, Dunitz J, Rubins HB, Maddaus MA, Niewoehner DE. Serum carcinoembryonic antigen as an adjunct to preoperative staging of lung cancer. J Thorac Cardiovasc Surg 1998;116:412-6.

25. Stokes TC, Stevens JF, Long P, Lockey E, Miller AL. Preoperative carcinoembryonic antigen and survival after resection of lung cancer. Br J Dis Chest 1980;74:390-4.

26. Daly BD, Mueller JD, Faling LJ, Diehl JT, Bankoff MS, Karp DD, et al. N2 lung cancer: outcome in patients with false-negative computed tomographic scans of the chest. J Thorac Cardiovasc Surg 1993;105:904-11.

27. Cybulsky IJ, Lanza LA, Ryan MB, Putnam JB Jr, McMurtrey MM, Roth JA. Prognostic significance of computed tomography in resected N2 lung cancer. Ann Thorac Surg 1992;54:533-7.

28. Phillips WC, Scott JA, Blasczcynski G. Statistics for diagnostic procedures. I. How sensitive is "sensitivity"; how specific is "specificity"? AJR Am J Roentgenol 1983;140:1265-70. 